PART IV

\author{
OBSERVATIONS OF \\ MAGNETIC FIELDS ASSOCIATED WITH \\ FLARES AND OTHER TRANSITORY PHENOMENA
}




\title{
SOLAR MAGNETIC FIELDS IN ASSOCIATION WITH FLARES
}

\author{
R. MICHARD \\ Observatoire de Paris-Meudon, France
}

\begin{abstract}
The association of $\mathrm{H} \alpha$ flares with photospheric magnetic field patterns is reviewed on the basis of empirical evidence. The insertion of flares in the $\mathbf{H}_{\|}$and $\mathbf{H}_{\perp}$ patterns is described. Present data on the evolution of magnetic structures in connection with flares suggest that flares result from special trends in this evolution leading to stressed and/or 'misconnected' fields. However no sudden changes with a flare-like time scale have been definitely observed in photospheric fields.
\end{abstract}

\section{The Insertion of Flares in Magnetic Patterns}

\subsection{OCCURRENCE OF FLARES AGAINST 'MAGNETIC TYPE' OF A.R.}

As is well known, flares (and flare-like transient events) are associated with Active Regions of we define as such an area of the Sun permeated by significant magnetic fields. This is even the case for the faint flare-like brightenings which follow the blow-off of quiescent filaments, since these filaments are separating extended regions of opposite magnetic polarities, resulting from the decay of the strong fields of Active Centers.

However, not all magnetic field patterns are likely to become the seat of a flare event. For a flare to occur the magnetic field must deviate somehow from the simple bipolar structure, characterized by two magnetic poles at the photosphere aligned roughly along the $E-W$ directon, with the line of separation of polarities, that is the zero line of $H_{V}$ (for vertical) not much inclined to the solar meridian.

Magnetic patterns departing to various degrees from the simple bipolar one are characterized in such empirical classifications as

- the classical Mt Wilson magnetic types of sunspots groups.

- the Meudon classification of $H_{\|}$patterns in A.R. (Martres et al., 1966a).

- the similar Mount Wilson classification (Smith and Howard, 1968).

These last two systems take into account the fields outside spots and lead to very good correlations with flare productivity.

\subsection{LOCATION OF FLARES RELATIVE TO THE INVERSIONS OF POLARITIES}

In complex or anomalous patterns of the magnetic field, the flares cannot occur at any place. We must qualify this statement, by saying that the initial brightenings in $H \alpha$ at the start of a flare have privileged positions. As early as 1958, Severny (1958) showed these to occur only at or very close to a line of polarity inversion, provided that the horizontal gradient of the longitudinal field across the inversion line was strong enough. He gave (Severny, 1960) the condition

$$
\Delta H_{\|} / \Delta x \gtrsim 0.1 \mathrm{G} / \mathrm{km} \text {. }
$$

Shortly after Michard et al. (1961) pointed out that this condition means in practice the presence of a strong horizontal field at the inversion line, ruling out any special 
association of flares with neutral lines or neutral points, at least at the photospheric level. The Crimean observers confirmed this in detail when they started observing transverse fields (Severny, 1961). On the other hand they showed a quantitative correlation between the strength of the local gradient of $H_{\|}$and the importance of the associated flare (Gopasyuk et al., 1963).

The condition about the strength of the horizontal gradient of $\mathrm{H}_{\|}$or equivalently about the strength of the transverse field at the photospheric level is by no means sufficient for the occurrence of flares (nor perhaps very strictly necessary).

Other suggestive properties of flare localization in connection with inversion lines of $\mathrm{H}_{\|}$have been described by Martres et al., 1966 and independently or subsequently by many observers (Moreton and Severny, 1966, 1968; Smith and Ramsey, 1967).

(1) In a perturbated bipolar structure having a main 'normal' inversion line separating the $E$ and $W$ polarities and some 'abnormal' inversion line, this latter one is the preferred seat of the flares. 'Abnormal' inversion lines may encircle an island or peninsula of following polarity inside the preceding one, or the reverse. Their essential property is to have the wrong orientation to the meridian, near $90^{\circ}$ rather than near $0^{\circ}$.

(2) The initial $\mathrm{H} \alpha$ brightenings tend to occur close to the inversion line rather than directly on it. In the case of flares showing more than one bright knot, that is the most frequent case, these knots are located on two different polarities, on both side of the inversion line. When bright elongated features develop, they do it along the inversion line on both sides of it.

The large proton flares with two bright ribbons overlying row spots of opposite polarities are a particular case of this general phenomenon.

It is interesting to note that the first observation of the X-ray emission of a flare, obtained at good spatial resolution by Vaiana et al., 1968, reveal a close correlation with the $\mathrm{H} \alpha$ emission. A two-ribbon structure is observed, with a bright cloud connecting the two ribbons above the inversion line.

\subsection{ASSOCIATION OF FLARES WITH HORIZONTAL (TRANSVERSE) FIELDS}

Besides the already mentioned result of the localization of flares at places of strong horizontal field in the photosphere, I should recall two interesting findings of the Crimean observers.

Severny $(1963,1964)$ studied the place of flares on maps showing the azimuth $\varphi$ of the transverse $\mathrm{H}_{\perp}$ component projected on the photosphere. He found that, as a rule, flares occur in regions of 'bifurcation' of $H_{\perp}$ : this means regions where the azimuth $\varphi$ changes abruptly from point to point indicating the proximity or perhaps the crossing of flux tubes with strongly different orientations. He also presented many cases where the horizontal field in a flare productive area runs nearly parallel to the inversion line, instead of crossing it frankly in the direction of the gradient of $\mathrm{H}_{\|}$. In such cases the flare ribbons may be said to extend parallel to the inversion line or to the horizontal field indifferently.

Besides direct polarimetric measurements, further indications on the orientations of 
transverse fields may be derived from the examination of elongated $\mathrm{H} \alpha$ structures, fibrils and small filaments, on high resolution pictures. The available descriptions are often consistent with the idea that the tubes of force in flare productive area, cross the inversion line at small angles and show erratic angular changes or 'bifurcations'.

From complete observations of the 3 components of the magnetic field, it is possible to compute the vertical component of the electric current

$$
j_{z}=\frac{1}{4 \pi}(\nabla \times \mathbf{H})_{z} .
$$

Severny found the location of a flare to correspond with the regions of maximum $j_{z}$. I shall not develop this point since you will hear a communication from Severny on these topics.

\subsection{CONCLUSIVE REMARKS}

To sum up, the empirical evidence suggests that the locus of flares are systems of tubes of force which, instead of connecting most directly the available magnetic poles, are badly elongated and stressed. Following the expression of Gold (1968), there are probably 'misconnections' in the magnetic field. Such a situation is obviously forced by the subphotospheric distribution of magnetic poles and, as a result, the field high above the photosphere may contain much more energy than a potential field.

\section{Evolution of Flare Productive Magnetic Patterns}

Changes of field structure in connection with flares have been a controversial subject and still are in some respects. In this section we shall talk about the less controversial part of the subject. Everybody agrees that flare productive Active Regions are progressively but fastly evolving; significant changes in spot morphology and magnetic structure may be observed in time intervals of the order of one hour. Various workers associated the productivity of flares with specific periods in the life of A.R. when significant evolution takes place (Bumba et al., 1968).

Obviously the building up of flaring magnetic structures is the result of an evolution in which take part the following processes:- Arrival of new poles and magnetic ropes from subphotospheric layers, often resulting in the birth of new spots. - Random surface motion of magnetic poles probably resulting from large scale convection. Differential rotation.

If we try to describe this complex evolution by some global parameters calculated over the whole A.R. such as spot area (Sivarenam, 1969; Sawyer, 1967), total flux of following and preceeding polarity $F_{f}$ and $F_{p}$, their sum or differences (Martres et al., 1967), and then to associate the changes of such parameters to the occurrence of flares, the results are often quite unclear as might be expected. However in connection with very large flares, spectacular changes of $F_{f}+F_{p}$ and $F_{f}-F_{p}$ have been recorded by Severny.

In general one should look in more detail to trace the changes possibly connected 


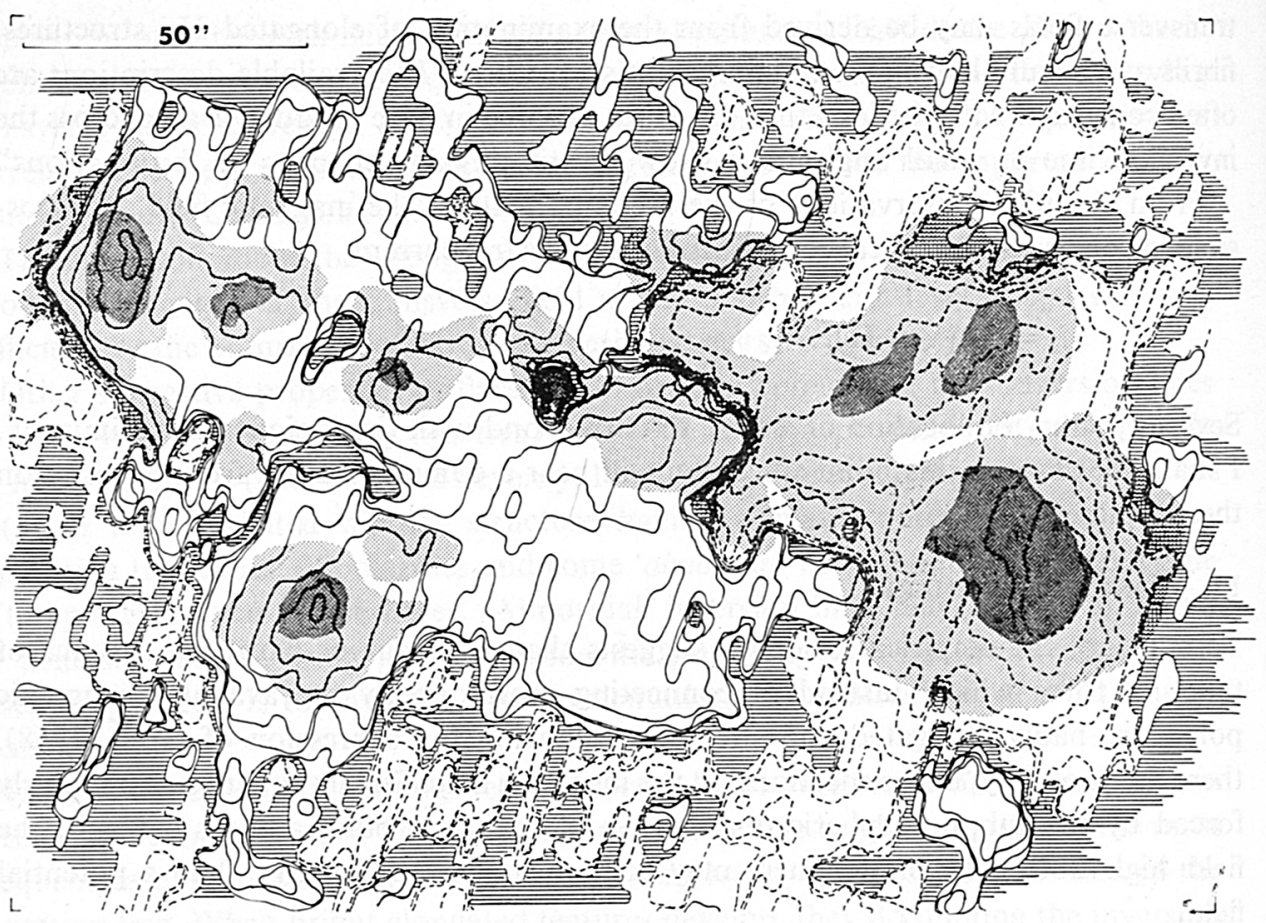

Fig. 1a.

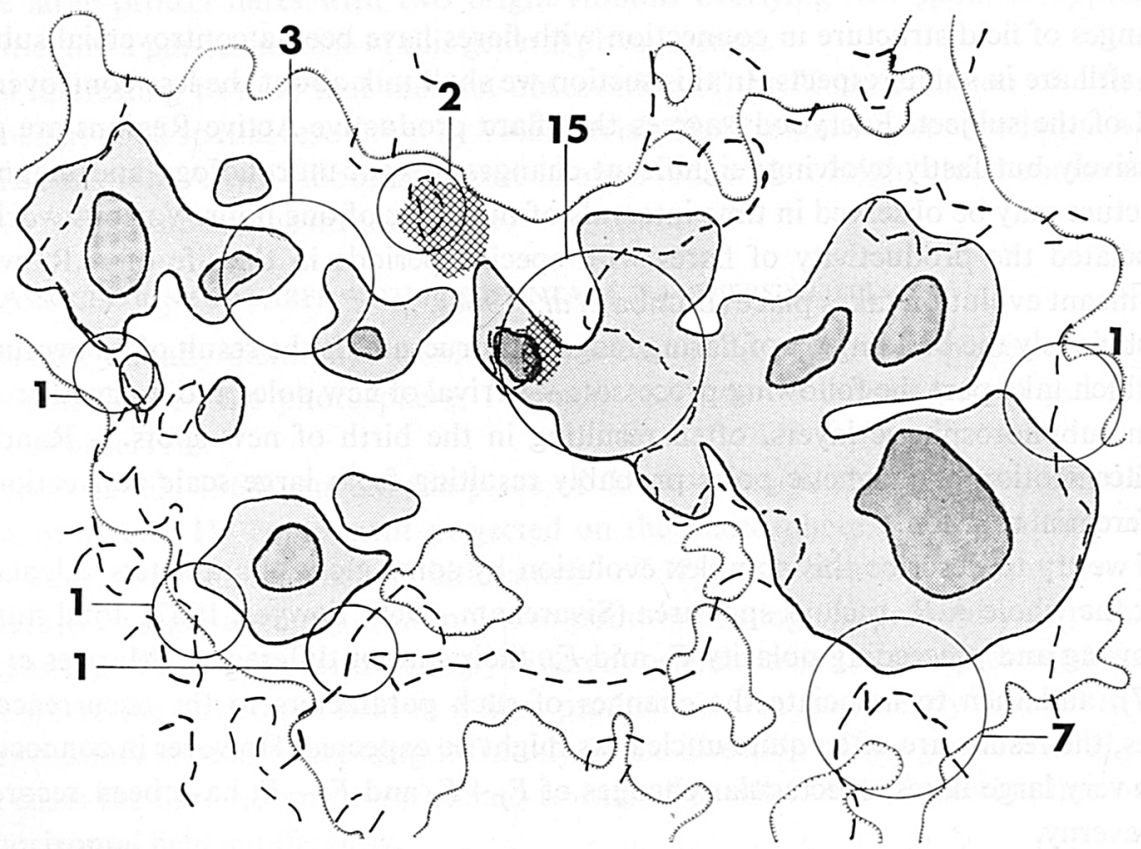

Fig. 1b. 
with flares. This was done first by Severny who compared magnetic maps taken a few hours apart in the best cases. He observed after flares a decrease of the gradient $\Delta \mathrm{H}_{\|} / \Delta x$ across the inversion line which has been the seat of the flare. Sometimes a magnetic hill involved in the flare has moved away from the inversion line, or has decreased or even disappeared (Severny, 1963).

Other observers have collected results which are more or less consistent with Severny's (Michard et al., 1961 ; Rust, 1968). However the word of caution of Michard et al., 1961 is still in order: the time distribution of observations is such that it impossible to tell if the observed changes are progressive, or sudden and coincident with the flare. Their internal accuracy also should be questioned, particularly for the measurement of horizontal gradients which are very sensitive to image quality.

In the case of very big flares, particularly proton flares, the Crimean observers (Gopasyuk et al., 1963; Severny, 1963; Howard and Severny, 1963) found very large changes in spot morphology and magnetic fluxes when comparing data taken a few hours before and after the event. For instance in the case of the cosmic ray flare of July 16, 1959 a decrease of all large umbral fields in the active center by a factor of 3 was found. However at Mount Wilson (Howard and Babcock, 1960) no flare associated change was noted in the smaller range of fields recorded (saturation at about $100 \mathrm{G})$. This different behavior is surprising.

In contradiction also with Severny, Malville and Tandberg-Hanssen (1969), found only minor changes when studying the evolution of the longitudinal field (measured in $H \alpha$ ) in connection with major flares of May 21-23, 1967, including a proton flare*.

In analysing data from the CSSAR, 1965, Martres et al. (1968a, b), found an interesting law of evolution of magnetic patterns involved in flares. These authors divide each complex pattern of longitudinal field into a number of 'Evolving Magnetic Features' or 'Structures Magnétiques Evolutives'. These features are local peaks of $\mathrm{H}_{\|}$, spotted or unspotted, of characteristic dimensions $10^{4}-10^{5} \mathrm{~km}$, each of a single polarity. The evolution of the whole pattern of course results from the individual stories of each EMF.

In accordance with previous results, each flare involves at least two adjacent EMF of opposite polarities. But it is found that these two EMF have opposite senses of evolution in the period of flare occurrence, one being increasing, the other decreasing.

* This paper also contains the first observation of magnetic fields in the flare plasma emitting $\mathrm{H} \alpha$.

Fig. 1. The location of a series of minor flares in a longitudinal magnetic field pattern. (a) Longitudinal field of an active center $24^{\circ} \mathrm{N}$ and $17^{\circ} \mathrm{E}$ on July 27 th, 1967 , observed at Meudon, time 08.50 UT. North is towards the top; east towards the left. Isogauss are for 15, 30, 50, 100, 250, 500, 1000, 1500 G ; horizontally hatched areas have fields smaller than $15 \mathrm{G}$. Major sunspots are also drawn and shaded from a $K_{1}$ spectroheliogram. The active center shows a number of 'inclusions' or 'parasitic polarities'. (b) On the same drawing of spots are shown the inversion lines and the regions of flare production during the same day from Quarterly Bulletin on Solar Activity: the observed number of flares and subfiares at each location is noted. Hatched: starting points of a flare of importance in, time 08.15-09.16, from an $\mathrm{H} \alpha$ film obtained in Meudon. This flare is one of several homologous flares at exactly the same location. Note that a few flares occurred on a zero of $\mathbf{H}_{\|}$without observed inversion of polarity. 
In the first work the evolution of EMF was characterized by the changes in area of the included spots. More recently, Ribes (1969) using a good series of maps of $\mathbf{H}_{\|}$of a single region taken at hourly intervals for three days (night excluded) could characterize each feature $A$ by its contribution to the flux integral $\Delta F=\int_{A} \mathrm{H} d s$ and was able to fully confirm the above law. In both work it was proven that if two adjacent EMF of opposite polarities have the same sense of variation, no flare can connect them.

It should be remarked that this law of variation of the magnetic features involved in flares, was established by the observers at Meudon on a sample of active regions producing only minor flares. This law is not in contradiction with the type of changes described by Severny and co-workers for active centers producing major flares, such as the decrease or disappearance of parts of the magnetic poles. However there is perhaps a difference in interpretation because we consider these changes as progressive. At least we do not believe that the accuracy of the available observations allows one to pin point sudden changes in direct coincidence with flares.

One may try to summarize as follows a typical evolution of flare productive magnetic patterns: these patterns contain several magnetic poles, of which two at least, being of opposite polarities, are evolving in opposite senses: the flux of pole $I$ through the photosphere increases, while the flux of pole $D$ decreases probably as a result of large scale convection. As a consequence part of the tube of forces connecting $I$ and $D$ are pulled and distorted: in the photosphere the lines of force are dragged with the high density plasma; in the chromosphere-corona however the re-arrangement of the tubes of force proceeds by sudden 'disconnections', i.e. flares.

However stresses of the magnetic ropes leading to preflare condition may also very well occur by horizontal motion of the poles (Gopasyuk et al., 1963) or by rotation (Gopasyuk, 1965; Stenflo, 1969).

\section{The Case for Sudden Changes of Magnetic Fields in the Photosphere in Correlation with Flares}

I have presented the empirical data as being consistent with progressive changes of the magnetic patterns in the photosphere, inducing 'catastrophic' changes in the chromosphere-corona. We must recall here that we are dealing with a complex situation where:

- in the upper solar atmosphere $\quad B^{2} / 8 \pi \gg \varrho v^{2}$

- in the deep photosphere $\quad B^{2} / 8 \pi \ll \varrho v^{2}$

the magnetic observations being available only for the 'boundary' between these two regions.

If we accept that a substantial part of the flare energy is derived from the magnetic energy in the upper layer, large changes of the coronal magnetic structure are necessary which should perhaps be reflected by significant changes at the photosphere, in close time association. For instance according to many authors, the energy of a large flare is $\gtrsim 10^{32} \mathrm{ergs}$; on the other hand the total magnetic energy of a large 
active center (taken on a thickness of $1000 \mathrm{~km}$ ) is of this same order of magnitude or lower. This, by the way, suggests that we should be less generous in our estimates of flare energy output, or give up the idea to pump it out of the magnetic field.

To come back to our subject we ask what is the evidence for abrupt changes of the photospheric field in close time association with flares? In my opinion the evidence is not conclusive from the magnetic observations alone, because they were not obtained with a sufficient resolution in time (only one map per hour in the best cases) and the effect of changes in seeing could not be checked.

Observations of the changes in photospheric structures which are closely correlated with magnetic fields, such as sunspots, did not disclose significant changes. On the other hand, chromospheric and coronal structures (including fine structure) are well known to present spectacular variations at the time of flares (Bruzek, 1968a, b; Bruzek and DeMastus, 1970; Wild, 1969).

Thus for the time being it is best to conclude that sudden changes of photospheric fields have not yet been definitely observed, although they probably exist since abrupt variations of the coronal fields should have some counterpart in the photosphere.

In conclusion this graph indicates the probable causal relations connecting the different classes of phenomena under discussion.

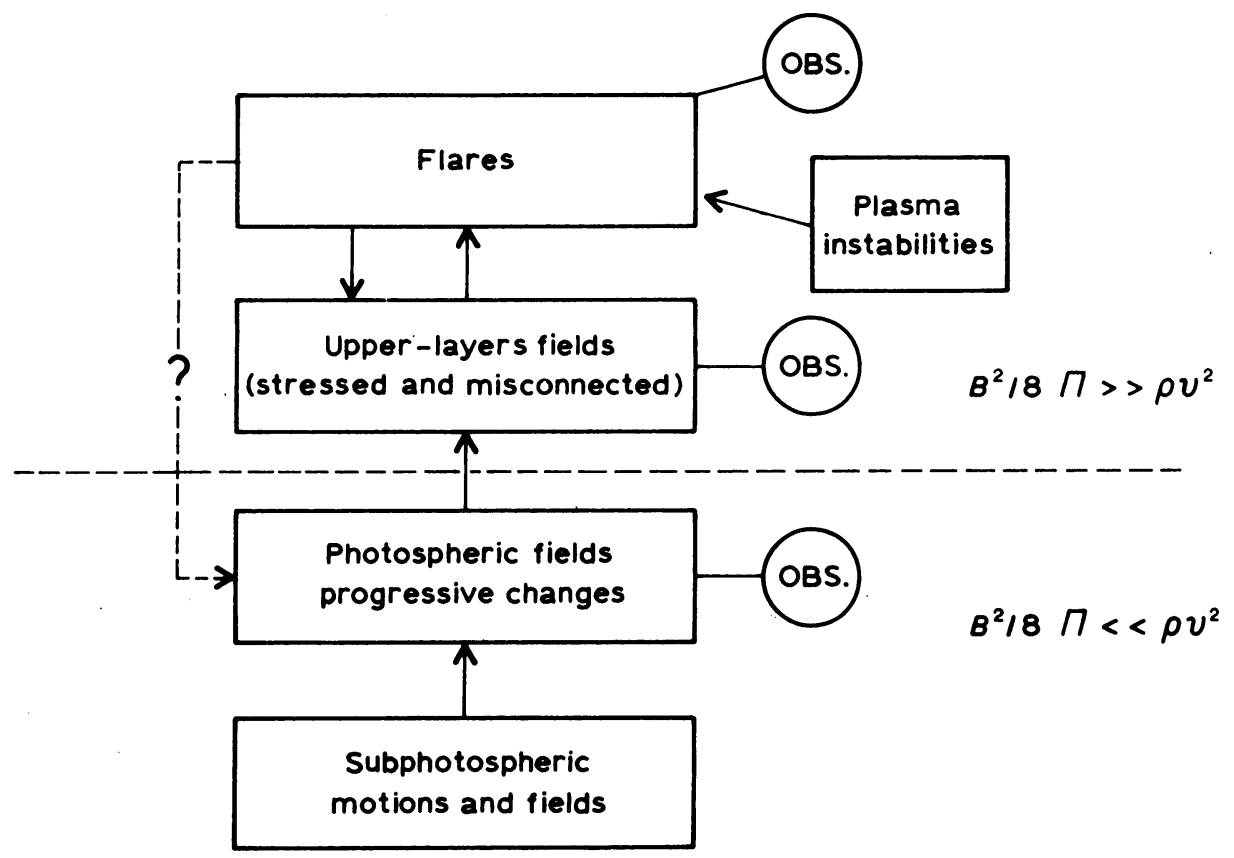

\section{References}

Bruzek, A. and DeMastus, H. W.: 1970, Solar Phys. $12,447$.

Bumba, V., Krivsky, L., Martres, M.-J., and Soru-Iscovici, I.: 1968, in K. O. Kiepenheuer (ed.), 'Structure and Development of Solar Active Regions', IAU Symp. 35, 311. 
Gold, T.: 1968, in Y. Öhman (ed.), 'Mass Motion in Solar Flares and Related Phenomena', Nobel Symp. 9, 205.

Gopasyuk, S. I., Ogir, M. B., Severny, A. B., and Shaposhnikova, E. F.: 1963, Izv. Krymsk. Astrofiz. Obs. 29, 15.

Gopasyuk, S. I.: 1965, Izv. Krymsk. Astrofiz. Obs. 33, 100.

Howard, R. and Babcock, H. W.: 1960, Astrophys. J. 132, 218.

Howard, R. and Severny, A. B.: 1963, Astrophys. J. 137, 1242.

Martres, M.-J., Michard, R., and Soru-Iscovici, I.: 1966a, Ann. Astrophys. 29, 245.

Martres, M.-J., Michard, R., and Soru-Iscovici, I.: 1966b, Ann. Astrophys. 29, 249.

Martres, M.-J., Michard, R., Soru-Iscovici, I. and Tsap, T.: 1968a, in K. O. Kiepenheuer (ed.), 'Structure and Development of Solar Active Regions', IAU Symp. 35, 318.

Martres, M.-J., Michard, R., Soru-Iscovici, I. and Tsap, T.: 1968b, Solar Phys. 5, 187.

McKim, Malville, J. and Tandberg-Hanssen, E.: 1969, Solar Phys. 6, 278.

Michard, R., Mouradian, Z., and Semel, M.: 1961, Ann. Astrophys. $24,54$.

Moreton, G. and Severny, A. B.: 1966, Astron. J. 71, 172.

Moreton, G. and Severny, A. B.: 1968, Solar Phys. 3, 282.

Ribes, E.: 1969, Astron. Astrophys. $2,316$.

Rust, D. M.: 1968, in K. O. Kiepenheuer (ed.), 'Structure and Development od Solar Active Regions', IAU Symp. 35, 77.

Severny, A. B.: 1958, Izv. Krymsk. Astrofiz. Obs. $20,22$.

Severny, A. B.: 1960, Izv. Krymsk. Astrofiz. Obs. 22, 12.

Severny, A. B.: 1963, in R. Lüst (ed.), 'Stellar and Solar Magnetic Fields', IAU Symp. 22, 238.

Severny, A. B.: 1964, Izv. Krymsk. Astrofiz. Obs. 31, 159.

Severny, A. B.: 1969, in Z. Švestka (ed.), 'The Proton Flare Project', Ann. IQSY 3, MIT Press, Cambridge, Mass., p. 11.

Sivaraman, K. R.: 1969, Solar Phys. 6, 152.

Smith, S. F. and Howard, R.: 1968, in K. O. Kiepenheuer (ed.), 'Structure and Development of Solar Active Regions', IAU Symp. 35, 33.

Smith, S. F. and Ramsey, H.: 1967, Solar Phys. $2,158$.

Stenflo, J. O.: 1969, Solar Phys. 8, 115.

Vaiana, G. S., Reidy, W. P., Zehnpfenning, I., Van Speybroeck, L., and Giacconi, R.: 1968, Science 161, 564.

Wild, J. P.: 1969, Solar Phys. 9, 260.

\section{Discussion}

Title: The gradient of a vertical field is about $1000 \mathrm{G} / \mathrm{s}$. The gradient of a horizontal field is of the same order. Since measurements of energy depend quadratically on $B$, errors of seeing can cause energy errors of orders of magnitudes.

Sweet: In correlating the $\mathrm{H} \alpha$ emission with the photospheric field it should be remembered that the $\mathrm{H} \alpha$ emission may not be occurring at the seat of the energy release. A better correlation may be forthcoming with the coronal fields when these can be measured.

Brueckner: From a XUV spectrum obtained during a rocket flight we, at the Naval Research Laboratory, think that the main source of the flare energy is a very small volume, in this case given by the resolution of the spectroheliograph, which was approximately 5 arc s. From the Stark effect splitting in the HeII line 304 and from the occurrence of the continuum, extremely high electron densities in this central part of the flare can be derived, but the interpretation of the spectra is still uncertain.

Dodson-Prince: Although it is true that most flares occur in centers of activity with well developed spots, strong magnetic fields, and steep gradients in the magnetic field, it seems appropriate to keep in mind the fact that approximately $7 \%$ of flares of importance $\geqslant 2$ have taken place in regions with either no spots or only very small spots with area of the order of 100 millionths of the hemisphere. The magnetic fields associated with these flares appear to be primarily the bipolar fields of old plages. These rare flares in regions without large spots indicate that at least occasionally very simple magnetic circumstances may accompany the development of major $\mathrm{H} \alpha$ flares. 